\title{
OPEN A spiking neural network (SNN) for detecting high frequency oscillations (HFOs) in the intraoperative ECoG
}

\author{
Karla Burelo ${ }^{1,2}$, Mohammadali Sharifshazileh ${ }^{1,2}$, Niklaus Krayenbühl $\mathbf{l}^{3,4}$, \\ Georgia Ramantani ${ }^{3,4,5}$, Giacomo Indiveri ${ }^{1,5}$ \& Johannes Sarnthein ${ }^{2,4,5}$
}

To achieve seizure freedom, epilepsy surgery requires the complete resection of the epileptogenic brain tissue. In intraoperative electrocorticography (ECOG) recordings, high frequency oscillations (HFOs) generated by epileptogenic tissue can be used to tailor the resection margin. However, automatic detection of HFOs in real-time remains an open challenge. Here we present a spiking neural network (SNN) for automatic HFO detection that is optimally suited for neuromorphic hardware implementation. We trained the SNN to detect HFO signals measured from intraoperative ECoG on-line, using an independently labeled dataset (58 $\mathrm{min}, 16$ recordings). We targeted the detection of HFOs in the fast ripple frequency range $(250-500 \mathrm{~Hz}$ ) and compared the network results with the labeled HFO data. We endowed the SNN with a novel artifact rejection mechanism to suppress sharp transients and demonstrate its effectiveness on the ECoG dataset. The HFO rates (median 6.6 HFO/ min in pre-resection recordings) detected by this $\mathrm{SNN}$ are comparable to those published in the dataset (Spearman's $\rho=0.81$ ). The postsurgical seizure outcome was "predicted" with $100 \%$ (CI [63 $100 \%]$ ) accuracy for all 8 patients. These results provide a further step towards the construction of a real-time portable battery-operated HFO detection system that can be used during epilepsy surgery to guide the resection of the epileptogenic zone.

Among patients with epilepsy, one-third have seizures that cannot be controlled by medication ${ }^{1}$. Selected patients with drug-resistant focal epilepsy may benefit from epilepsy surgery to achieve seizure freedom. The efficacy of epilepsy surgery requires the complete resection of the epileptogenic brain tissue ${ }^{2}$. Intraoperative electrocorticography $(\mathrm{ECoG})$ can be performed during surgery to optimize the delineation of the epileptogenic zone (EZ) against healthy brain tissue by taking into account interictal spike patterns ${ }^{3-6}$. This so called "tailoring" may guide surgical decisions, but the value of interictal spikes as an epilepsy biomarker in this context is under debate ${ }^{7}$.

Interictal high frequency oscillations (HFOs $>80 \mathrm{~Hz}$ ), particularly in the fast ripple band $(250-500 \mathrm{~Hz}$ ), are being discussed as biomarkers to guide surgical decisions. In intraoperative ECoG, the primary purpose of HFO detection is the delineation of the EZ, i.e. the prediction of recurrent seizures if the EZ has not been entirely resected $^{7-16}$. As a fundamental problem, HFOs have been defined non-uniformly across research studies ${ }^{7}$. For example, in one definition of $\mathrm{HFO}^{17}$, HFO locations varied across hours or days in intracranial electroencephalography (iEEG) during sleep in most patients. In contrast, in another definition of $\mathrm{HFO}^{18}$, the test-retest reliability of HFO locations in iEEG was high over hours or days in those patients where HFOs were highly predictive of seizure outcome $\mathrm{e}^{10,19}$; this latter definition of HFO is therefore of high clinical relevance.

HFO detection faces the challenge of low signal-to-noise ratio, which can be improved by high density electrodes $^{8,20}$ and by low-noise amplification ${ }^{11}$. As a further challenge, a clinically relevant HFO must be distinguished from the electrical artifacts induced by the standard intraoperative devices or any other spurious oscillation in the fast ripple band. To achieve clinical relevance, an HFO should (1) be defined prospectively and (2) be validated against postsurgical seizure freedom ${ }^{21}$. While there are many automated detection schemes that define HFOs prospectively, only few validated the detected HFOs against postsurgical seizure freedom ${ }^{7-13}$.

\footnotetext{
${ }^{1}$ Institute of Neuroinformatics, University of Zurich and ETH Zurich, 8057 Zurich, Switzerland. ${ }^{2}$ Department of Neurosurgery, University Hospital Zurich, University of Zurich, 8091 Zurich, Switzerland. ${ }^{3}$ University Children's Hospital, University of Zurich, 8032 Zurich, Switzerland. ${ }^{4}$ Klinisches Neurozentrum Zürich, University Hospital Zurich, University of Zurich, 8006 Zurich, Switzerland. ${ }^{5}$ Neuroscience Center Zurich, ETH Zurich, 8092 Zurich, Switzerland. ${ }^{\square}$ email: johannes.sarnthein@usz.ch
} 
These detectors however, require further offline processing of the pre-recorded signal to apply an automatic or semiautomatic artifact rejection stage to eliminate events wrongly classified as HFO. This offline processing requirement limits the possibility to perform real-time detection of HFOs during the time-span available within the constraints of the surgery.

These constraints have hampered the application of HFO in clinical practice. Also, clinical trials are yet missing. Currently, a first small study is expected to report results on the non-inferiority of HFOs compared to spikes $^{14}$. Ultimately, the value of HFO for delineating the EZ must be confirmed in a large prospective clinical trial with a large numbers of patients recruited from multiple centers. Standardized HFO analysis in multiple centers will need fast, reliable, and unsupervised automatic HFO detection. Further, a prospective definition of a clinically relevant HFO will have to be agreed on. In earlier work, we have used intraoperative ECoG data ${ }^{15}$ to establish an automated definition of clinically relevant HFOs that predicts postoperative seizure freedom ("Spectrum detector") $)^{9,22}$. The HFOs of the Spectrum detector have since been validated to predict seizure freedom in independently recorded datasets ${ }^{8,11}$.

Here, we simulated a spiking neural network (SNN) for HFO detection in the intraoperative ECoG. This work builds on a previously validated SNN for HFO detection in the intracranial EEG (iEEG) ${ }^{23,24}$, and extends it by introducing a novel artifact rejection mechanism to reject fast transient artifacts, and by validating it on intraoperative electrocorticography recordings (ECoG). As a computational principle, the SNN emulates the spiking of neurons in small networks ${ }^{25}$ so that they can be implemented in low-power and compact neuromorphic hardware that perform real-time computation ${ }^{26}$.

We applied our SNN to a published dataset of pre-recorded ECoG, where HFOs were detected offline using the Spectrum detector ${ }^{9,22}$ and validated against postoperative seizure freedom ${ }^{8}$. In this benchmark testing, we were able to correctly predict the postoperative seizure outcome in all 8 patients. This is a further step towards an SNN that may be implemented in a neuromorphic device ${ }^{23}$ for standardized and real-time HFO detection during epilepsy surgery.

\section{Methods}

Patients. We retrospectively included pediatric and adult patients (median age 18.5 years, range [12, 33 ] years) who (1) underwent epilepsy surgery in our institutions, (2) where the resection was guided by intraoperative high-density ECoG (hd-ECoG), (3) post-resection hd-ECoG was available, and (4) the follow-up-duration after surgery was $\geq 12$ months.

The patients were followed-up at the outpatient clinics of Neurosurgery, University Hospital Zurich, and Epileptology, University Childrens Hospital Zurich, 3, 6, 12, 24 months after surgery according to our institutional protocol. Postsurgical seizure outcome was determined according to the International League Against Epilepsy (ILAE) scale and consecutively classified in two categories: seizure freedom (ILAE 1) and seizure recurrence (ILAE 2-5).

Ethical considerations. The collection of personal patient data and their analysis were approved and performed conform to the guidelines and regulations of the local research ethics committee (Kantonale Ethikkommission Zürich KEK-ZH-Nr. 2019-01977), who waived the collection of patients' written informed consent.

Anesthesia management. According to our standard protocol for neurosurgical interventions, anesthesia was induced with intravenous application of Propofol $(1.5-2 \mathrm{mg} / \mathrm{kg})$ and Fentanyl $(2-3 \mu \mathrm{g} / \mathrm{kg})$. Intratracheal intubation was facilitated by Atracurium $(0.5 \mathrm{mg} / \mathrm{kg})$. Anesthesia was maintained with Propofol $(5-10 \mathrm{mg} / \mathrm{kg} / \mathrm{h})$ and Remifentanil $(0.1-2 \mu \mathrm{g} / \mathrm{kg} / \mathrm{min})$. Twenty minutes before ECoG recording, Propofol was ceased and anesthesia was sustained with Sevoflurane $(\mathrm{MAC}<0.5)$.

ECoG recordings. Intraoperative ECoG was recorded with high-density subdural grid electrodes (hdECoG, AdTech Medical, contact exposure diameter $2.3 \mathrm{~mm}$, inter-electrode distance $5 \mathrm{~mm}$ ). We used a needle electrode placed in the dura as electrical reference. We collected ECoG data with a Nicolet recording device (Nicolet CSeries amplifier: Natus Medical Incorporated, 16-bit ADC, Pleasanton, PA, USA; sampling rate $2 \mathrm{kHz}$, 1-800 Hz passband). All ECoG data was re-referenced to a bipolar montage along the length of the grid. During preprocessing, we selected intervals of stable recordings. Channels affected by continuous interference or not recording from brain tissue were excluded from further analysis. The placement of subdural electrodes for intraoperative ECoG was guided solely by the clinical question. Only interictal epileptiform discharges (and not HFO) were considered for the intraoperative delineation of the EZ and thus for tailoring the resection. In this study, we first analysed the ECoG recorded from a location over the volume to be resected and its margins (pre-resection ECoG), and then the post-resection ECoG that was recorded at the resection margins. The neurosurgeon and the neurologist in charge of the patient carefully documented the ECoG electrode localization with respect to the resected volume. This data is publicly available as described earlier ${ }^{8}$ and can be found at https:// gin.g-node.org/USZ_NCH/Intraoperative_ECoG_HFO.

HFO detection with the Spectrum detector. The Spectrum detector has been described in detail in previous publications $s^{8,9,11,22}$. In brief, the detector has three stages. Stage I determined a baseline amplitude threshold in time intervals with high Stockwell entropy (low oscillatory activity). Events exceeding the threshold were marked as events of interest (EoI). In Stage II, the detector selected all EoI that exhibited a high frequency peak isolated from low frequency activity in the time-frequency space ${ }^{22}$. The number of EoI was further reduced in Stage III, where artifacts with multichannel spread were rejected, since HFO are spatially confined in a small patch of cortical tissue ${ }^{20,27}$. Following these steps of automated HFO detection, an observer inspected the events 
a

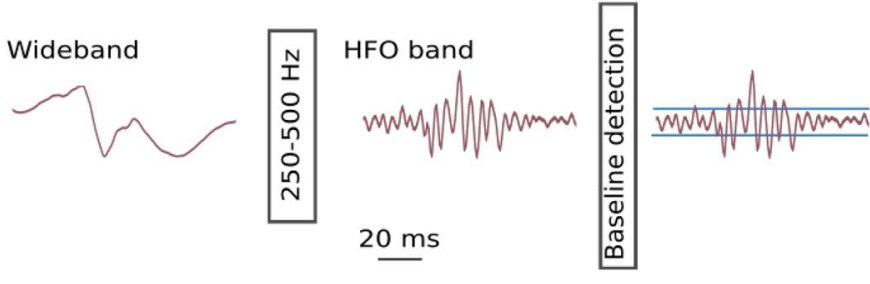

c

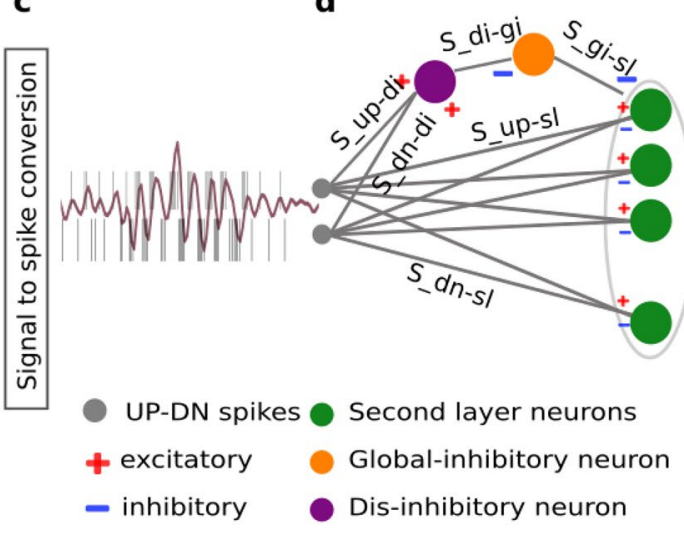

Figure 1. HFO detection scheme. (a) The wideband ECoG is filtered in the HFO frequency band (250-500 $\mathrm{Hz}$ ). (b) In the baseline detection stage, the background noise of the signal is used to set the signal-to-spike threshold. (c) The signal-to-spike conversion algorithm converts the analog signal into two streams of digital outputs: UP and DN spikes. (d) The SNN architecture for HFO detection and artifact rejection consists of input neurons (grey) receiving the input UP-DN spikes. These inputs project to a second layer of neurons (green) and to a dis-inhibitory neuron (purple). This neuron projects inhibitory synapses to a global-inhibitory neuron (orange), which is continuously inhibiting the second layer neurons. The synapses of the projections are excitatory (positive, red) or inhibitory (negative, blue).

\begin{tabular}{|l|l|l|l|l|}
\hline Connection & Name & Connection strength (fA) & Polarity & Time constant $\boldsymbol{\tau}$ (ms) \\
\hline Input UP spikes to second layer & $S_{u p-s l}$ & {$\left[\begin{array}{ll}714\end{array}\right]$} & exc & {$[36]$} \\
\hline Input DN spikes to second layer & $S_{d n-s l}$ & {$[714]$} & inh & $\tau_{S_{u p-s l}}[0.11]$ \\
\hline Input UP spikes to dis-inhibitory neuron & $S_{u p-d i}$ & 21 & exc & 5 \\
\hline Input DN spikes to dis-inhibitory neuron & $S_{d n-d i}$ & 21 & exc & 5 \\
\hline Dis-inhibitory neuron to global-inhibitory neuron & $S_{d i-g i}$ & 17.5 & inh & 20 \\
\hline Poisson to global-inhibitory neuron & $S_{p o i s s-g i}$ & - & exc & 5 \\
\hline Global-inhibitory neuron to second layer & $S_{g i-s l}$ & 24.5 & inh & 5 \\
\hline
\end{tabular}

Table 1. Synapse parameters of the SNN detector. A connection between two neurons (Fig. 1d) is characterized by the positive (excitatory, exc) or negative (inhibitory, inh) current in fA and the time constant.

\begin{tabular}{|c|c|c|c|c|c|c|c|c|c|c|}
\hline \multirow[b]{3}{*}{ Patient } & \multirow[b]{3}{*}{ Etiology } & \multirow[b]{3}{*}{ Resection area } & \multirow{3}{*}{$\begin{array}{l}\text { Follow-up } \\
\text { (months) }\end{array}$} & \multirow{3}{*}{\begin{tabular}{|l|} 
Seizure \\
Outcome \\
(ILEA) \\
\end{tabular}} & \multirow{2}{*}{\multicolumn{2}{|c|}{\begin{tabular}{|l|} 
Spectrum \\
HFO rate \\
\end{tabular}}} & \multirow{2}{*}{\multicolumn{2}{|c|}{\begin{tabular}{|l|} 
SNN \\
HFO rate \\
\end{tabular}}} & \multirow{3}{*}{\begin{tabular}{|l|} 
Spectrum \\
Outcome \\
Prediction \\
\end{tabular}} & \multirow{3}{*}{\begin{tabular}{|l|} 
SNN \\
Outcome \\
Prediction \\
\end{tabular}} \\
\hline & & & & & & & & & & \\
\hline & & & & & Pre & Post & Pre & Post & & \\
\hline 1 & DNET & L medial parietal & 33 & 1 & 6 & $<1$ & 3 & $<1$ & TN & $\mathrm{TN}$ \\
\hline 2 & FCD 2b & L dorsal medial prefrontal & 24 & 1 & 4 & $<1$ & 10 & $<1$ & $\mathrm{TN}$ & TN \\
\hline 3 & Sturge Weber & L lateral occipital & 30 & 1 & 2 & $<1$ & 1 & $<1$ & TN & TN \\
\hline 4 & Ganglioglioma & R post. fusiform gyrus (occipital) & 18 & 1 & 8 & $<1$ & 12 & $<1$ & $\mathrm{TN}$ & $\mathrm{TN}$ \\
\hline 5 & FCD 2a & R post. temporal & 13 & 1 & 13 & $<1$ & 30 & $<1$ & TN & TN \\
\hline 6 & Sturge Weber & R lateral post. temporal \& lateral occipital & 20 & 3 & 32 & 5 & 45 & 14 & TP & TP \\
\hline 7 & Astrocytoma & R post. middle frontal gyrus & 29 & 1 & 1 & $<1$ & 1 & $<1$ & TN & TN \\
\hline 8 & FCD 2a & R post. cingulate gyrus, post. hippocampus & 12 & 1 & 22 & $<1$ & 2 & $<1$ & TN & TN \\
\hline
\end{tabular}

Table 2. Patient characteristics, HFO rates and postsurgical seizure outcome. We present the maximum HFO rates $(\mathrm{HFO} / \mathrm{min})$ in the pre- and post-resection ECoG as detected by the Spectrum detector ${ }^{8}$ and the SNN detector. We "predict" seizure outcome for each patient based on whether remaining HFOs were observed in the post-resection recordings or not. We compare the "prediction" with the seizure outcome (ILAE). FCD focal cortical dysplacia, DNET dysembryoplastic neuroepithelial tumors, $R$ right, $L$ left, post. posterior, $T P$ true positive, $T N$ true negative. 
in wideband and filtered in the HFO band to reject further artifacts. This prospective definition of a clinically relevant HFO has been shown to predict postsurgical seizure outcome with high accuracy ${ }^{8,9,11}$.

HFO detection with the SNN. As a first step in the HFO detection pipeline, the wideband ECoG recording was filtered in the $250-500 \mathrm{~Hz}$ fast ripple band (Fig. 1a). Since Butterworth filters showed to be a good approximation of the Tow-Thomas architectures for the hardware SNN filters ${ }^{23,24,28}$, we used a 2 nd order filter of this model and the SciPy Python package to simulate the filtering stage ${ }^{29-31}$. In the filtered signal, we defined a baseline amplitude that has to be exceeded by a putative HFO event (Fig. 1b). Following the algorithm implemented in the hardware $\mathrm{SNN}^{23}$, we selected a $1 \mathrm{~s}$ time window, stored the maximum signal amplitudes of consecutive non-overlapping time windows of $50 \mathrm{~ms}$, and took the mean of the lowest quartile as the baseline amplitude.

The filtered signal was then converted to spikes using a delta conversion scheme (Fig. 1c) that is inspired by the analog delta modulator $(\mathrm{ADM})^{32,33}$. The $\mathrm{ADM}$ integrates an error signal which follows the input until it increases above (decreases below) the upper (lower) threshold. Upon the crossover, an UP (DN) spike is generated and the error will reset to zero. The error will remain zero for a refractory period after which, it will continue tracking the input again until the next threshold crossover, hence encoding the input into UP-DN spike trains. This threshold was set at $50 \%$ of the pre-recorded ECoG baseline amplitude. To approximate the asynchronous conversion of the signal in the ADM, we over-sampled the input signal at $35 \mathrm{kHz}$ and we set a refractory period $(300 \mu \mathrm{s})$ to simulate the delay of the ADM after each spike. Note that the over-sampling period should be much smaller than the refractory period for accurate data conversion.

The HFO detection stage of the network (the core SNN) consists of input neurons receiving the input UP-DN spikes and a second layer of neurons (Fig. 1d). The projections to the second layer neurons are excitatory for UP spikes and inhibitory for DN spikes. We used the Python SNN simulator Brian2 ${ }^{34}$, the custom toolbox Teili ${ }^{35}$, and the parameters in Table 1 to simulate an SNN that matches the behavior of the neuromorphic circuits of the hardware $\mathrm{SNN}^{23}$. The software simulations take into account the neuromorphic circuit properties. As the circuits are based on a "current-mode" design, we represented all the relevant state variables (such as the neuron membrane potential) as currents (e.g., $\left.I_{m e m}\right)$.

Spikes in the second layer neurons were used to mark an HFO. Any spike within a $15 \mathrm{~ms}$ window indicated an HFO, where consecutive windows containing spikes were concatenated to indicate the same HFO. There were no further steps required for artifact rejection. HFO detection was performed independently for each channel of the pre- and post-resection ECoG recordings.

Prediction of seizure outcome using residual HFO. The output of the HFO detection was compared to the postsurgical seizure outcome in each patient. For each patient, we calculated the HFO rate in each electrode channel of the pre- and post-resection recordings by dividing the number of HFOs detected in the channel by the duration of the recording. We compared the HFO rate between pre- and post- resection recordings by only selecting the recording channel that had the highest HFO rate (Table 2), since the presence of a single channel with residual HFO has shown to predict seizure recurrence ${ }^{8,9,11,12,14-16}$. Channels with HFO rates of $\geq 1 \mathrm{HFO} /$ $\mathrm{min}$ in the last post-resection $\mathrm{ECoG}$ were defined as having residual $\mathrm{HFOs}^{8,9}$.

To quantify the predictive value of HFO with respect to seizure outcome in each patient, we retrospectively "predicted" seizure freedom (ILAE 1) in patients with post-resection $\mathrm{HFO}$ rates of $<1 \mathrm{HFO} / \mathrm{min}$ and recurrent seizures (ILAE 2-6) in patients with post-resection HFO rates of $\geq 1 \mathrm{HFO} / \mathrm{min}$ (Table 2). We divided the patients into four groups. True Positive (TP): residual HFO, seizure recurrence (ILAE >1) correctly predicted; True Negative (TN): no residual HFO, seizure freedom (ILAE 1) correctly predicted; False Positive (FP): residual HFO, seizure freedom falsely predicted; False Negative (FN): no residual HFO, seizure recurrence falsely predicted. The positive predictive value was calculated as $\mathrm{PPV}=\mathrm{TP} /(\mathrm{TP}+\mathrm{FP})$, negative predictive value as $\mathrm{NPV}=\mathrm{TN} /$ $(\mathrm{TN}+\mathrm{FN})$, sensitivity as Sens $=\mathrm{TP} /(\mathrm{TP}+\mathrm{FN})$, specificity as Spec $=\mathrm{TN} /(\mathrm{TN}+\mathrm{FP})$, and accuracy as $\mathrm{ACC}=$ $(\mathrm{TP}+\mathrm{TN}) /(\mathrm{TP}+\mathrm{TN}+\mathrm{FP}+\mathrm{FN})$.

Statistics. For statistical testing, we used non-parametric methods. To compare two distributions, we used the Wilcoxon Rank Sum test. To assess the relationship between two variables, we used the Spearman Rank Correlation coefficient $\rho$. We calculated the $95 \%$ confidence interval (CI) of a ratio on the basis of the binomial distribution. Statistical significance was established at $\mathrm{p}<.05$.

\section{Results}

Architecture of the core SNN augmented by the artifact rejection stage. As a first result, we describe here the architecture of the SNN that is augmented by the artifact rejection stage (Fig. 1d). For the core SNN, we used a two-layered feed-forward network of integrate and fire neurons with dynamic synapses ${ }^{23}$. For the first layer of the network, we used two input neurons that projected UP-DN spike trains to the second layer neurons using excitatory and inhibitory synapses, respectively. As for the artifact rejection stage, we implemented a dis-inhibitory mechanism using a dis-inhibitory neuron and an global-inhibitory neuron that constantly suppressed the activity of the second layer neurons. For this purpose, the global-inhibitory neuron was stimulated with a Poisson spike train to generate continuous spikes at $135 \mathrm{~Hz}$. The dis-inhibitory neuron projected inhibitory synapses to the global-inhibitory neuron and received the input UP-DN spikes through excitatory synapses. 
a

High Frequency Oscillation

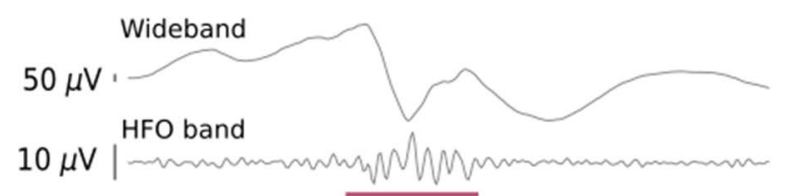

$\begin{array}{ll}\widehat{N} & 700 \\ \underbrace{}_{4} & 400 \\ & 100\end{array}$

Spikes

\section{UP | | | |||||||IIIIII||}

DN || |

b
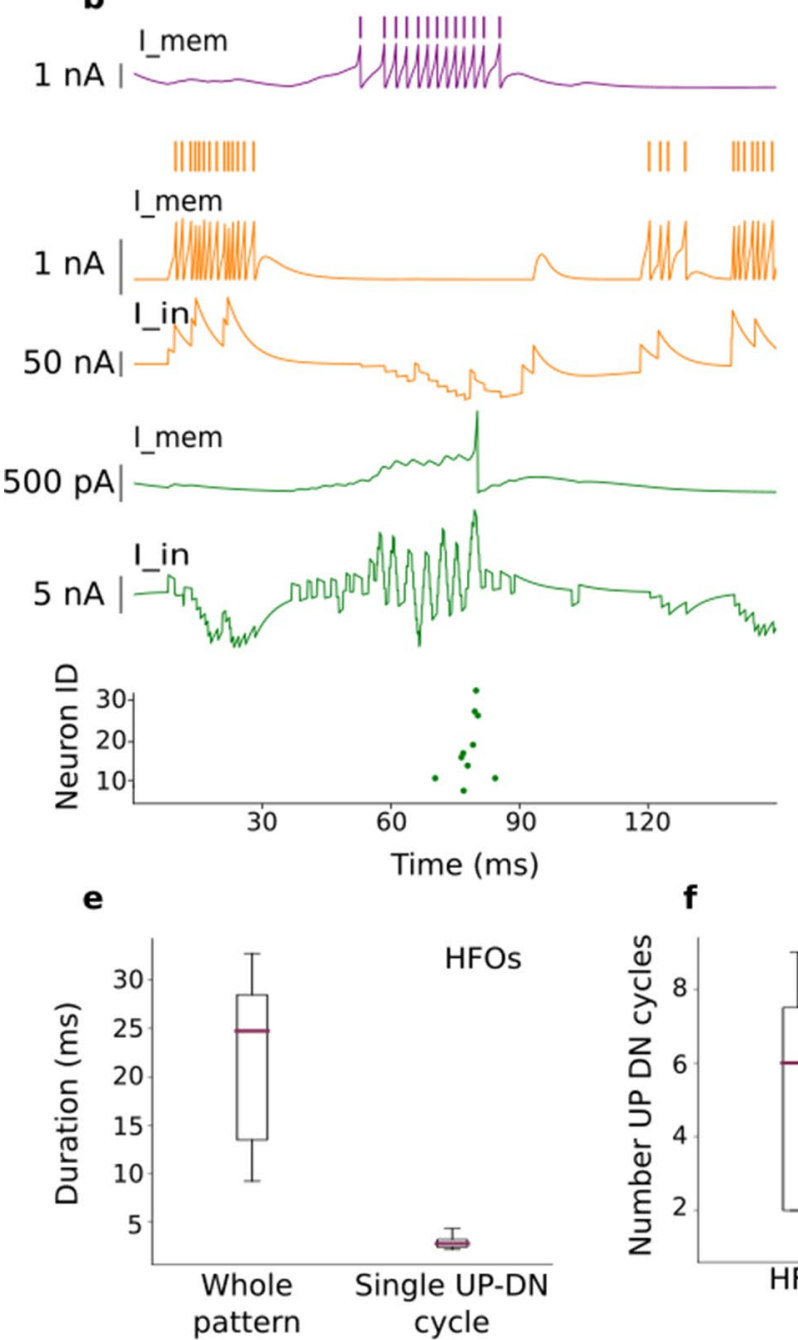

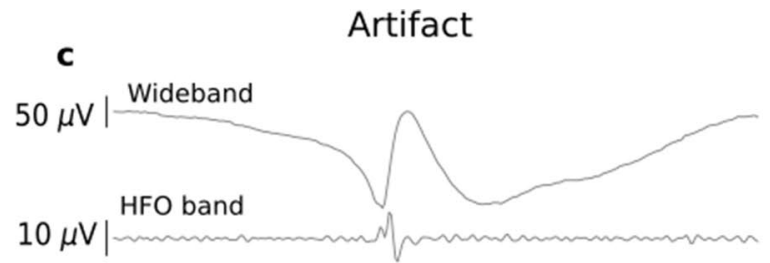

$\left.\begin{array}{ll}\widehat{N} & 700 \\ \underbrace{}_{4} & 400 \\ & 100\end{array}\right]$

Spikes

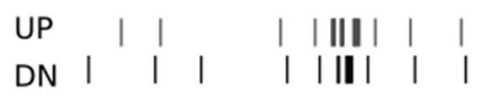

d
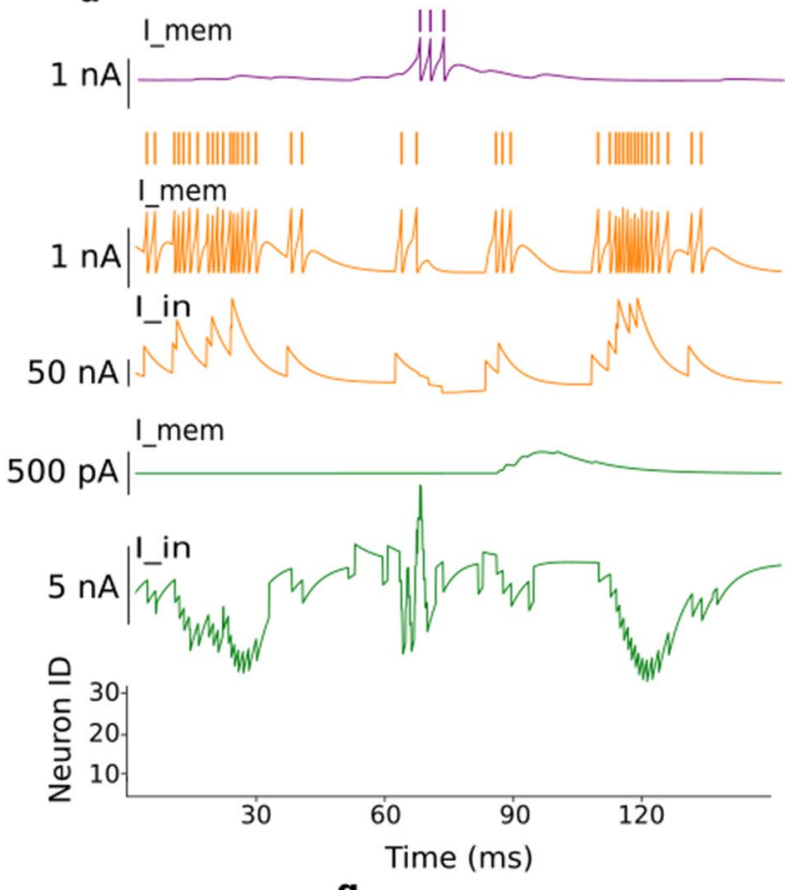

g

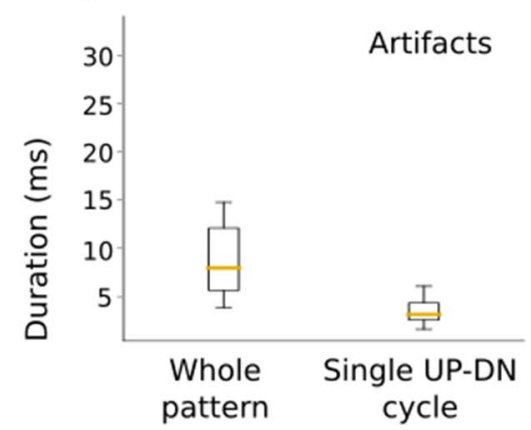

Figure 2. The SNN distinguishes between an HFO and an artifact (fast transient) in the ECoG. (a,c) ECoG signal and input spike train to the SNN. Consecutive UP-DN spike bursts in the train define an UP-DN cycle. More cycles occurred during the HFO. (b,d) Activity of the neurons in the SNN. (b) During an HFO, the input spike train excited the dis-inhibitory neuron (membrane potential $I_{m e m}$ in purple), which suppressed the global-inhibitory neuron that became silent (input current $I_{i n}$ and $I_{m e m}$ in orange). A neuron of the core SNN (input current $I_{i n}$ and membrane potential $I_{m e m}$ in green) integrated the input spike trains and produced an output spike. Several second layer neurons of the core SNN responded to the HFO (green dots). (d) During a short fast transient in the ECoG, the excitation of the dis-inhibitory neuron was so short (purple trace) that it did not silence the global-inhibitory neuron (orange traces), which in turn continued to inhibit the second layer neuron (green traces) and prevented the generation of an output spike. Neither this nor any other neuron of the second layer $I_{m e m}$ (green) reached the spiking threshold hence, the raster plot remained empty. (e,f,g) Population characteristics of the UP-DN input spike trains entering the second layer neurons and the dis-inhibitory neuron. (e) The train during an HFO lasted $24 \mathrm{~ms}$ (median), with a single cycle lasting $2.6 \mathrm{~ms}$ (median). (f) The HFOs comprise more UP-DN cycles (median 6 cycles) than the artifacts (median 2 cycles). (g) The train during an artifact lasted 9 ms (median), with a single cycle lasting $3.2 \mathrm{~ms}$ (median). The cycle characteristics were used to select the parameters of the dis-inhibitory neuron and the global-inhibitory neuron (Table 1). 
Parameters for the artifact rejection stage. To calibrate the artifact rejection stage, the optimal parameters were found heuristically by analysing HFOs and sharp transients in the ECoG as follows.

We composed a training signal of 22 snippets ( $50 \mathrm{~ms}$ wide) and fed it into the core $\mathrm{SNN}$, i.e. the SNN without the artifact rejection stage. We selected 11 snippets where an HFO had been marked by both the core SNN and the Spectrum detector ${ }^{8}$, as published together with the dataset ${ }^{36}$. Further, we selected a representative set of 11 snippets where the core SNN had marked an HFO but the Spectrum detector had not marked an HFO, i.e. a snippet that the core SNN had wrongly classified as HFO. These snippets contained sharp transients that (after filtering) can have similar waveforms to HFOs (Fig. 2c).

The signals were then transformed in UP-DN spike trains (Fig. 2a,c).In a spike train, we defined as an "UPDN cycle" a burst of UP spikes followed by a burst of DN spikes. From the spike trains we quantified (1) the duration of the spike train, (2) the number of cycles and (3) the inter-spike-interval (ISI) within the bursts of a cycle. We observed that fast transients generated two cycles (median) in the UP-DN spike train while HFOs generated more cycles (median 6 cycles, Fig. 2f).

To inhibit the second layer neurons during a fast transient, the global-inhibitory neuron must suppress their activity for at least one cycle. Conversely, to dis-inhibit the activity of the second layer neurons during an HFO, the dis-inhibition should start as early as possible (i.e. after the first cycle) and should last the total duration of the HFO.

In our data set, the median HFO duration (24 ms) was longer than the duration of an artifact (8 ms) (Fig. 2e,g, $\mathrm{p}<.008$ Wilcoxon Rank Sum test). Nevertheless, the median duration of a single cycle during a fast transient was larger $(3.2 \mathrm{~ms})$ than a single cycle during an HFO $(2.6 \mathrm{~ms})$ (Fig. 2e,g, p<.008). Also the median number of cycles differed between HFOs and artifacts (Fig. 2f, 6 vs. 2, p<.011).

Therefore, the suppression of the activity of the second layer neurons during the first cycle resulted in a suppression of HFOs with short duration. However, this design choice did not affect the maximum HFO rates in the benchmarking between our SNN detector and the Spectrum detector (Table 2).

Since the dis-inhibitory neuron received excitatory inputs from both UP and DN spikes, any activity in the signal could have caused the activation of the dis-inhibitory neuron and, in consequence, the dis-inhibition of the second-layer neurons and an erroneous HFO detection. We avoided this dis-inhibition by using a short synaptic time constant for the connections of the dis-inhibitory neuron. Hence, the dis-inhibitory neuron was activated only during periods of elevated UP-DN spiking as it occurred during a fast transient or an HFO.

Example of a detected HFO and a suppressed transient. The SNN activity differed markedly between detection of an HFO and rejection of a fast transient (Fig. 2 b,d). The UP-DN spike train generated spikes in the membrane potential of the dis-inhibitory neuron (purple trace $I_{m e m}$ ). Note that one UP-DN cycle has passed until the dis-inhibitory neuron responded to the UP-DN inputs. Only after this delay of one cycle, this neuron inhibited the global-inhibitory neuron (orange $I_{i n}$ trace). Note that this trace results from integrating the inputs from the Poisson spike train and the inhibitory inputs from the dis-inhibitory neuron.

During the presence of an HFO in the signal, the global-inhibitory neuron remained silent ( $\sim 30 \mathrm{~ms}$, purple bar in Panel a). Meanwhile, the neuron in the second layer integrated the UP-DN spikes in its input signal (green $I_{\text {in }}$ trace Panel b) and accumulated enough evidence in its membrane potential (green $I_{m e m}$ trace) to generate a spike in response to the HFO. The raster plot on the bottom of Panel b shows the spikes of other neurons in the second layer that also responded to this HFO. Detection of an HFO was defined as a spike of at least one second layer neuron.

During the presence of a fast transient in the signal, all the neurons in the second layer remained silent as seen in the raster plot in the bottom of Panel d. Similarly as in the HFO example, once one UP-DN cycle has passed, the dis-inhibitory neuron responded to the UP-DN inputs. However, its activation was not sufficient to suppress the activity of the global-inhibitory neuron. This neuron was active during the presence of the transient (orange traces) and inhibited the second layer neuron. The second layer neuron integrated the UP-DN spikes, which increased the green input current $I_{\text {in }}$. However, before it could accumulate enough evidence, the neuron was inhibited by the global-inhibitory neuron which kept this neuron and the whole second layer silent. Thus, the dis-inhibitory neuron was activated only when an HFO was present in the signal; fast transients in the ECoG were suppressed in the SNN and not misclassified as HFO.

The HFO rates from SNN and spectrum detector are comparable. For each patient, we counted the number of HFOs detected per electrode channel and divided by the duration of the recording (median 3.5 $\mathrm{min}$, total data duration $58 \mathrm{~min}$ ) to obtain the HFO rate (Table 2). We found maximal HFO rates $\geq 1 \mathrm{HFO} / \mathrm{min}$ in the pre-resection recordings of all 8 patients ( 8 recordings, median duration $3.9 \mathrm{~min}$, median $6.6 \mathrm{HFO} / \mathrm{min}$, range [1.3-45.0] HFO/min). The HFO rates of the Spectrum and the SNN detector were correlated $(\rho=.81, \mathrm{p}$ $<.0001$ Spearman Rank Correlation). In total, the SNN and the Spectrum detector found 4293 HFOs and 2336 HFOs, respectively. While we do not aim for a one-to-one correspondence between detected HFO events, the performance of the new SNN detector is comparable to that of the well-established Spectrum detector.

Residual HFO predict poor seizure outcome. We used the presence of a single channel containing HFOs in the post-resection ECoG to predict seizure recurrence ${ }^{15,16}$ In the post-resection recordings ( 8 recordings, median duration $3.0 \mathrm{~min}$ ), seven of the 8 patients had $<1 \mathrm{HFO} / \mathrm{min}$, i.e. there were no residual HFOs. Given the absence of residual HFOs, we "predicted" good postsurgical seizure outcome in these patients. Indeed, these 7 patients achieved seizure freedom after surgery (ILAE 1, median follow-up period after surgery 22 months). Only the post-resection recordings of Patient 6 showed an $\mathrm{HFO}$ rate $\geq 1 \mathrm{HFO} / \mathrm{min}$, which qualifies as residual HFOs. The presence of post-resection HFOs "predicted" poor surgical outcome in this patient who 
a

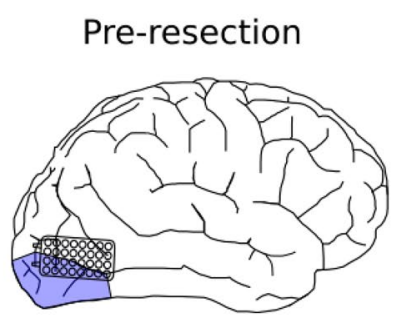

b

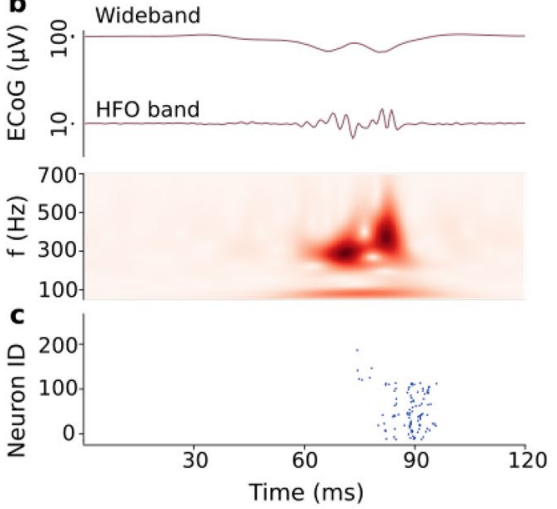

f

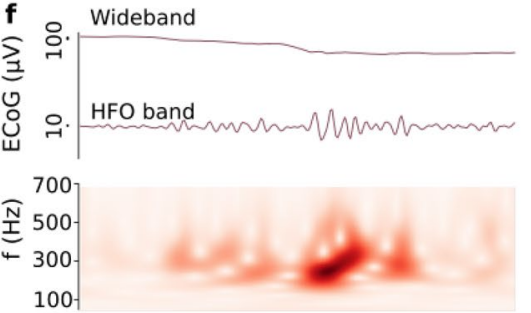

g

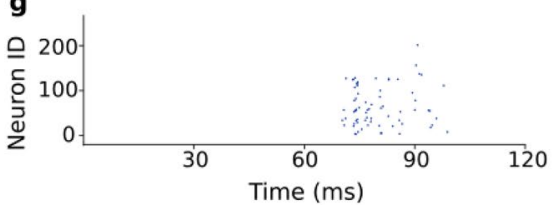

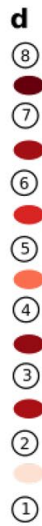

h

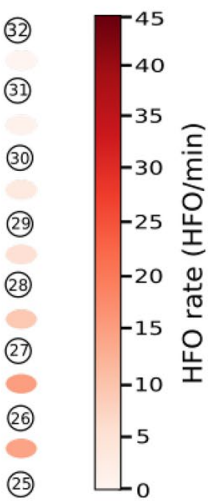

\begin{tabular}{|c|c|c|c|}
\hline (8) & (16) & (24) & (32) \\
\hline (7) & (15) & (23) & (31) \\
\hline (6) & (14) & (22) & (30) \\
\hline (5) & (13) & (21) & (29) \\
\hline (4) & (12) & (20) & (28) \\
\hline (3) & (11) & (19) & (27) \\
\hline (2) & (10) & (18) & (26) \\
\hline & (9) & (17) & (25) \\
\hline
\end{tabular}

Figure 3. Intraoperative ECoG recording and HFO detection in Patient 6. (a) Position of the high-density grid electrode before resection. (b) Example of an HFO in the pre-resection ECoG, wideband and filtered in HFO band $(250-500 \mathrm{~Hz})$, and the time-frequency spectrum. (c) Firing of SNN neurons indicate the occurrence of an HFO. (d) Pre-resection HFO rates for each recording channel. (e) Electrode position after resection. (f) Example of an HFO in the post-resection ECoG. (g) Firing of SNN neurons indicate the occurrence of an HFO. (h) Post-resection HFO rates for each recording channel. In 4 channels (28-29, 29-30, 30-31, 1-2), the HFO rate exceeded $1 \mathrm{HFO} / \mathrm{min}$. The occurrence of these residual HFOs "predict" poor seizure outcome. Indeed, the patient suffered from recurrent seizures (ILAE 3).

indeed suffered from recurrent seizures (ILAE 3). Fig. 3 shows the electrode placement, an example of a detected HFO by the SNN, and the HFO rates per recording channel for Patient 6 in both, pre- (Panels a-d) and postresection recordings (Panels $\mathrm{f}-\mathrm{j}$ ). As a cautionary note, the high rate of residual HFOs in the occipital cortex in Patient 6 (but neither in Patient 3 nor in Patient 4) might be related to the occurrence of physiological HFOs that are known to abound in the occipital lobe of the healthy brain ${ }^{37}$. Over the group of patients, the prediction accuracy was $100 \%$ (CI [63 100\%]).

\section{Discussion}

In this study, we simulated a new SNN that detects HFO in intraoperative ECoG. Compared to our previous $\mathrm{SNN}^{23}$, we have added an artifact rejection stage to suppress fast transients in the signal. Based on the HFO rates detected in the post-resection ECoG, we were able to "predict" the patients' seizure outcome with $100 \%$ accuracy. These results are preliminary evidence that the automatically detected HFOs by the SNN may indeed be clinically relevant.

Comparison with the spectrum detector. The ECoG recordings analysed here have been previously analysed for HFOs with the Spectrum detector ${ }^{8}$. The SNN detector in our study performed well while being fully unsupervised, which is an advantage for its possible application in multi-center studies. In designing the SNN, the aim was not to achieve a one-to-one agreement of the detected HFO events. Rather, the HFO rate threshold of $\geq 1 \mathrm{HFO} / \mathrm{min}$ in unresected channels was used to "predict" seizure recurrence. Both, our simulated SNN and the Spectrum detector reached the same "prediction" for each patient of this study (Table 2). Even though the SNN prediction of the poor outcome was limited to data from one patient, when not considering only post- 
resection but also pre-resection recordings, the SNN reached the same qualitative result (HFOs present / HFOs not present) in all 16 recordings.

As a difference to the SNN, the Spectrum detector applied multi-channel information to reject artifacts (Stage III) and, additionally, visual artifact rejection. These measures were required by the more challenging artifacts in the data recorded with $10 \mathrm{~mm}$ electrode distance. As a limitation, in our study we require the integration of densely spaced electrode contacts and low impedance to enable fully automated HFO detection with the SNN.

Clinical considerations. In our study, we analysed ECoG with a SNN detector that had been previously trained on an independent datase $\mathrm{t}^{23,38}$. The $100 \%$ outcome accuracy speaks for the robustness of automatically detected HFO to predict postoperative seizure freedom. Our study is limited by the small number of patients, where only one out of the eight patients had recurrent seizures. Our finding that residual HFO predicted seizure recurrence is in agreement with previous studies showing that incomplete resection of cortical tissue generating HFO correlates with seizure recurrence ${ }^{8,9,11,12,14-16}$.

Future implementation in neuromorphic hardware. In a previous study, we have designed a neuromorphic device ${ }^{23}$ to detect HFO in iEEG during deep sleep ${ }^{38}$. That device demonstrated that common pre-processing stages like low-noise amplification, filtering and signal transformation using ADMs can be implemented in the same silicon die alongside a multi-core neuromorphic processor to allow on-line and real-time postprocessing of biomedical signals. The device is compact, battery-powered and does not interfere with other electronic equipment, which would facilitate its use during surgery. Seizure outcome prediction on deep sleep iEEG was comparable with the outcome prediction achieved with an HFO detector based on template matching ${ }^{11}$.

In the current study, we have advanced the SNN presented in our earlier study ${ }^{23}$ for HFO detection in intraoperative recordings. Compared to deep sleep iEEG, the electronic interference from standard machinery in the operating theatre make HFO detection in the intraoperative ECoG more challenging. For example, the surgery environment induces fast transients in the wideband ECoG that appear as short oscillations in the HFO frequency range. Hence, for HFO detection in ECoG recordings, we have extended the SNN by adding an artifact rejection stage on top of the core SNN.

Obviously, our new simulated SNN was motivated by a future implementation in neuromorphic hardware ${ }^{39}$. The parameters of the pre-processing stages were applicable to both iEEG and ECoG and remained unchanged. In the same way, the core SNN performed equally well in the detection of clinically relevant HFO in both iEEG and ECoG with identical parameter settings. Also for the artifact rejection stage, all the architecture and parameter decisions have been chosen such that the simulated SNN can easily be implemented in the neuromorphic hardware with only slight adaptations.

\section{Conclusions}

To detect HFO in intraoperative ECoG, we simulated a SNN extended with an artifact rejection stage to suppress sharp transients. Confirming earlier results, the occurrence of post-resection HFO predicted seizure recurrence. This detector uses SNN computing and thereby is radically different from other detectors. These results provide a further step towards real-time detection of HFO during epilepsy surgery by an SNN implemented in neuromorphic hardware.

\section{Data availability}

The ECoG data and HFO markings are freely available at https://gin.g-node.org/USZ_NCH/Intraoperative ECoG_HFO. The code for the SNN detector is available at the GitHub repository https://github.com/kburel/ SNN_HFO_ECoG.

Received: 10 November 2020; Accepted: 5 March 2021

Published online: 24 March 2021

\section{References}

1. Ryvlin, P., Cross, J. H. \& Rheims, S. Epilepsy surgery in children and adults. Lancet Neurol. 13, 1114-1126 (2014).

2. Jette, N., Reid, A. Y. \& Wiebe, S. Surgical management of epilepsy. CMAJ 186, 997-1004 (2014).

3. Lesko, R. et al. The clinical utility of intraoperative electrocorticography in pediatric epilepsy surgical strategy and planning. $J$. Neurosurg. Pediatrics 1, 1-10 (2020).

4. Demuru, M. et al. The value of intra-operative electrographic biomarkers for tailoring during epilepsy surgery: From group-level to patient-level analysis. Sci. Rep. 10, 1-18 (2020).

5. Grewal, S. S. et al. Reassessing the impact of intraoperative electrocorticography on postoperative outcome of patients undergoing standard temporal lobectomy for mri-negative temporal lobe epilepsy. J. Neurosurg. 1, 1-10 (2019).

6. Gröppel, G. et al. Single stage epilepsy surgery in children and adolescents with focal cortical dysplasia type ii-prognostic value of the intraoperative electrocorticogram. Clin. Neurophysiol. 130, 20-24 (2019).

7. Chen, Z., Maturana, M. I., Burkitt, A. N., Cook, M. J. \& Grayden, D. B. High-frequency oscillations in epilepsy: What have we learned and what needs to be addressed. Neurology 96, 439-448 (2021).

8. Boran, E. et al. High-density ECoG improves the detection of high frequency oscillations that predict seizure outcome. Clin. Neurophysiol. 130, 1882-1888. https://doi.org/10.1016/j.clinph.2019.07.008 (2019).

9. Fedele, T. et al. Automatic detection of high frequency oscillations during epilepsy surgery predicts seizure outcome. Clin. Neurophysiol. 127, 3066-3074. https://doi.org/10.1016/j.clinph.2016.06.009 (2016).

10. Fedele, T. et al. High frequency oscillations detected in the intracranial EEG of epilepsy patients during interictal sleep, patients' electrode location and outcome of epilepsy surgery. Collaborative Res. Comput. Neurosci.https://doi.org/10.6080/K06Q1VD5 (2017).

11. Fedele, T. et al. Prediction of seizure outcome improved by fast ripples detected in low-noise intraoperative corticogram. Clin. Neurophysiol. 128, 1220-1226 (2017). 
12. Weiss, S. A. et al. Visually validated semi-automatic high-frequency oscillation detection aides the delineation of epileptogenic regions during intra-operative electrocorticography. Clin. Neurophysiol. 129, 2089-2098 (2018).

13. Wang, Y. et al. Expert consensus on clinical applications of high-frequency oscillations in epilepsy. Acta Epileptol. 2, 1-10 (2020).

14. van't Klooster, M. A. et al. High frequency oscillations in the intra-operative ECoG to guide epilepsy surgery ("the HFO trial"): Study protocol for a randomized controlled trial. Trials 16, 1-10 (2015).

15. van't Klooster, M. A. et al. Residual fast ripples in the intraoperative corticogram predict epilepsy surgery outcome. Neurology 85, $120-128(2015)$.

16. van't Klooster, M. A. et al. Tailoring epilepsy surgery with fast ripples in the intraoperative electrocorticogram. Ann. Neurol. 81, 664-676 (2017).

17. Gliske, S. V. et al. Variability in the location of high frequency oscillations during prolonged intracranial eeg recordings. Nat. Commun. 9, 1-14 (2018).

18. Burnos, S., Frauscher, B., Zelmann, R., Haegelen, C. \& Sarnthein, J. The morphology of high frequency oscillations (HFO) does not improve delineating the epileptogenic zone. Clin. Neurophysiol. 127, 2140-2148 (2016).

19. Dimakopoulos, V. et al. Blinded study: prospectively defined high frequency oscillations predict seizure outcome in individual patients (in review). medRxiv. https://doi.org/10.1101/2020.12.24.20248799 (2020). (submitted).

20. Zweiphenning, W. J. et al. The resolution revolution: Comparing spikes and high frequency oscillations in high-density and standard intra-operative electrocorticography of the same patient. Clin. Neurophysiol. 131, 1040 (2020).

21. Fedele, T., Ramantani, G. \& Sarnthein, J. High frequency oscillations as markers of epileptogenic tissue-end of the party?. Clin. Neurophysiol. 130, 624-626 (2019).

22. Burnos, S. et al. Human intracranial high frequency oscillations (HFOs) detected by automatic time-frequency analysis. PloS One 9, e94381. https://doi.org/10.1371/journal.pone.0094381 (2014).

23. Sharifhazileh, M., Burelo, K., Sarnthein, J. \& Indiveri, G. An electronic neuromorphic system for real-time detection of high frequency oscillations (HFOs) in intracranial EEG. arXiv preprint arXiv:2009.11245 (2020).

24. Sharifshazileh, M., Burelo, K., Fedele, T., Sarnthein, J. \& Indiveri, G. A neuromorphic device for detecting high-frequency oscillations in human iEEG. in IEEE International Conference on Electronics, Circuits and Systems (ICECS), 69-72 (IEEE, 2019).

25. Roy, K., Jaiswal, A. \& Panda, P. Towards spike-based machine intelligence with neuromorphic computing. Nature 575, 607-617 (2019).

26. Chicca, E., Stefanini, F., Bartolozzi, C. \& Indiveri, G. Neuromorphic electronic circuits for building autonomous cognitive systems. Proc. IEEE 102, 1367-1388 (2014).

27. Burnos, S., Fedele, T., Schmid, O., Krayenbühl, N. \& Sarnthein, J. Detectability of the somatosensory evoked high frequency oscillation (HFO) co-recorded by scalp EEG and ECoG under propofol. NeuroImage: Clin. 10, 318-325 (2016).

28. Fleischer, P. \& Tow, J. Design formulas for biquad active filters using three operational amplifiers. Proc. IEEE 61, 662-663 (1973).

29. Butterworth, S. et al. On the theory of filter amplifiers. Wirel. Eng. 7, 536-541 (1930).

30. Selesnick, I. W. \& Burrus, C. S. Generalized digital butterworth filter design. IEEE Trans. Signal Process. 46, 1688-1694 (1998).

31. Virtanen, P. et al. Scipy 1.0: fundamental algorithms for scientific computing in python. Nat. Methods 17, 261-272 (2020).

32. Yang, M., Liu, S.-C. \& Delbruck, T. A dynamic vision sensor with $1 \%$ temporal contrast sensitivity and in-pixel asynchronous delta modulator for event encoding. IEEE J. Solid-State Circuits 50, 2149-2160 (2015).

33. Corradi, F. \& Indiveri, G. A neuromorphic event-based neural recording system for smart brain-machine-interfaces. IEEE Trans. Biomed. Circuits Syst. 9, 699-709. https://doi.org/10.1109/TBCAS.2015.2479256 (2015).

34. Goodman, D. \& Brette, R. Brian: A simulator for spiking neural networks in Python. Front. Neuroinform. 2, https://doi.org/10. 3389/neuro.01.026.2009 (2008)

35. Milde, M. et al. Teili: a toolbox for building and testing neural algorithms and computational primitives using spiking neurons (2018). Unreleased software (University of Zurich and ETH Zurich, Institute of Neuroinformatics).

36. Boran, E. et al. Dataset of intraoperative pre- and post-resection ECoG recorded from epilepsy patients and fast ripple (FR) markings. g-node. https://gin.g-node.org/USZ_NCH/Intraoperative_ECoG_HFO (2019).

37. Frauscher, B. et al. High-frequency oscillations in the normal human brain. Ann. Neurol. 84, 374-385. https://doi.org/10.1002/ ana.25304 (2018).

38. Fedele, T. et al. Resection of high frequency oscillations predicts seizure outcome in the individual patient. Sci. Rep. 7, 13836. https://doi.org/10.1038/s41598-017-13064-1 (2017).

39. Borton, D. A., Dawes, H. E., Worrell, G. A., Starr, P. A. \& Denison, T. J. Developing collaborative platforms to advance neurotechnology and its translation. Neuron 108, 286-301 (2020).

\section{Acknowledgements}

This project has received funding from Swiss National Science Foundation (SNSF 320030_176222) and from the European Research Council (ERC) under the European Union's Horizon 2020 research and innovation program grant agreement No 724295 .

\section{Author contributions}

J.S. and G.I. conceived the experiments, N.K. and G.R. treated the patients, K.B. and M.S. conducted the experiments and analysed the results. K.B. and J.S. wrote the manuscript. All authors critically reviewed the manuscript.

\section{Competing interests}

The authors declare no competing interests.

\section{Additional information}

Correspondence and requests for materials should be addressed to J.S.

Reprints and permissions information is available at www.nature.com/reprints.

Publisher's note Springer Nature remains neutral with regard to jurisdictional claims in published maps and institutional affiliations. 
(c) (i) Open Access This article is licensed under a Creative Commons Attribution 4.0 International cc) License, which permits use, sharing, adaptation, distribution and reproduction in any medium or format, as long as you give appropriate credit to the original author(s) and the source, provide a link to the Creative Commons licence, and indicate if changes were made. The images or other third party material in this article are included in the article's Creative Commons licence, unless indicated otherwise in a credit line to the material. If material is not included in the article's Creative Commons licence and your intended use is not permitted by statutory regulation or exceeds the permitted use, you will need to obtain permission directly from the copyright holder. To view a copy of this licence, visit http://creativecommons.org/licenses/by/4.0/.

(C) The Author(s) 2021 